\title{
Effect of ethanol extracts of Antrodia cinnamomea on head and neck squamous cell carcinoma cell line
}

\author{
$\operatorname{Li~Liu(ii)}^{1 *}$ and Chen Wang (ii) ${ }^{2 *}$ \\ ${ }^{1}$ Anhui Medical College, Hefei, China \\ ${ }^{2}$ Fuyang Vocational Technical College, Fuyang, China
}

\begin{abstract}
Head and neck squamous cell carcinoma (HNSCC) is one of the most common malignant tumors. Ethanol extract of Antrodia cinnamomea (EEA) has been widely studied for its health benefits including anticancer effects. The purpose of this study was to assess the effects of EEA on HNSCC. Cell proliferation, transwell, and wound healing assays were performed. The impact of EEA on tumor growth was investigated using a xenograft model. Expressions of migration-related proteins (MMP-2, MMP-9, TIMP-1, and TIMP-2) and apoptosis-related proteins (cleaved caspase-9 and cleaved PARP) were determined using western blot analysis. The results indicated that EEA significantly inhibited the capacities of proliferation, invasion, and migration of HNSCC cells in a dose-dependent manner. Cleaved caspase- 9 and cleaved PARP expressions were increased in cells treated with an increasing concentration of EEA, which suggested that EEA induced apoptosis of HNSCC. MMP-2 and MMP-9 were downregulated when cells were administered EEA, while TIMP-1 and TIMP-2 were not affected, which uncovered the mechanisms mediating the EEAinduced inhibition on cell invasion and migration. The animal experiment also suggested that EEA inhibited tumor growth. Our study confirmed the inhibitive effects of EEA on cell proliferation, invasion, and migration of HNSCC in vitro and in vivo, providing the basis for further study of the application of EEA as an effective candidate for cancer treatment.
\end{abstract}

Key words: Antrodia cinnamomea; Head and neck squamous cell carcinoma cells; Proliferation; Invasion; Migration

\section{Introduction}

Head and neck squamous cell carcinoma (HNSCC) is one of the most common malignant tumors, representing over $95 \%$ of all head and neck cancer cases $(1,2)$. Despite advanced prevention and treatment strategies, thousands of new cases are reported annually (3), while treatment outcomes are still unsatisfactory with a five-year overall survival of about $50 \%$ (1). Most patients experience recurrence and poor prognosis after surgical resection, leading to a high mortality rate $(4,5)$. Studies have revealed that the high recurrence rate is mainly due to enhanced invasion, migration, and proliferation capacities of tumor cells (6). The development of new chemotherapeutic agents for HNSCC is urgently needed.

Antrodia cinnamomea has been commonly used as a traditional Taiwanese medicine for its diverse applications in healthcare including anticancer effects (7). Previous studies have established its anti-tumor effects $(1,8,9)$. The protective role of the ethanol extract of fruiting bodies of Antrodia cinnamomea has been experimentally demonstrated in several malignancies including renal cancer (10), lung cancer $(7,11)$, hepatocellular carcinoma (12), and breast cancer (13), while the effects on head and neck cancer were seldom evaluated (14). Several active components isolated from raw extracts of Antrodia cinnamomea, mainly including triterpenoids, dicarboxylic acids, and polysaccharides (15), have been identified and can effectively inhibit proliferation, invasion, and migration of tumor cells $(10,14)$. Moreover, extracts of Antrodia cinnamomea were reported to sensitize lung cancer cells and hepatic carcinoma cells to chemotherapeutics $(9,12,13,16,17)$, indicating it can serve as a promising source of adjuvant agents combined with conventional therapy. However the potential application of Antrodia cinnamomea in treatment of HNSCC remains unclear.

In this study, we aimed to assess the potential effect of Antrodia cinnamomea on HNSCC cells.

\section{Material and Methods}

\section{Ethanol extract of fruiting bodies of Antrodia cinnamomea (EEA)}

Fruiting bodies of Antrodia cinnamomea were purchased from Cosmox Biomedical Co. LTD (Taiwan) and identified by the Bioresource Collection and Research

Correspondence: Chen Wang: <wangchenbio@sina.com>

*Both authors contributed equally to this work. 
Center (BCRC, Taiwan). EEA was prepared as described in a previous study (7) with minor modifications. Briefly, $300 \mathrm{~g}$ of dried fruiting bodies of Antrodia cinnamomea was soaked in $1 \mathrm{~L}$ ethanol for three days at room temperature. Then, the sample was filtered with filter paper and the filtrate was collected. The residue was soaked in $1 \mathrm{~L}$ of fresh ethanol again, the sample was filtered, and the filtrate was collected. All filtrate was pooled and concentrated to a paste by vacuum distillation using a rotary evaporator (N-11, EYELA; Japan). The extract was dissolved in dimethylsulfoxide (DMSO, Sigma-Aldrich Corp., USA) at a concentration of $50 \mathrm{mg} / \mathrm{mL}$ as a stock solution for further study.

\section{Cell culture}

A tongue squamous cell carcinoma line (SAS) was purchased from the Type Culture Collection of the Chinese Academy of Sciences (China) and maintained in DMEM (HyClone, USA) supplemented with $10 \%$ fetal bovine serum (HyClone). Cells were cultured in a humidified incubator at $37^{\circ} \mathrm{C}$ under $5 \% \mathrm{CO}_{2}$.

\section{Western blot analysis}

Total cell protein was extracted using RIPA lysis buffer (Cell Signaling Technology, USA). Protein was determined by a Pierce BCA protein assay kit (Thermo Fisher Scientific, Inc., USA). Then, protein samples were resolved on $10 \%$ SDS-PAGE and transferred to polyvinylidene difluoride membranes. The membranes were blocked with $5 \%$ fat free milk for $1 \mathrm{~h}$ at room temperature, then incubated with the primary antibodies targeting human GAPDH (Cell Signaling Technology, Cat 8884), cleaved PARP (Cell Signaling Technology, Cat 5625), cleaved caspase-9 (Cell Signaling Technology, Cat 9505), MMP-2 (Cell Signaling Technology, Cat 40994), MMP-9 (Cell Signaling Technology, Cat 13667), TIMP-1 (Cell Signaling Technology, Cat 8946), or TIMP-2 (Cell Signaling Technology, Cat 5738) overnight at $4^{\circ} \mathrm{C}$. After washing, the membrane was incubated with goat anti-rabbit (Cell Signaling Technology, Cat 7074) or rabbit anti-mouse (Cell Signaling Technology, Cat 7076) lgG H\&L secondary antibody conjugated with horseradish peroxidase. Recommended dilutions for each antibody are listed in Table 1. The signal was developed using SuperSignal West Dura Extended Duration chemiluminescence substrate (Thermo Fisher Scientific, Inc.) and measured by ChemiDoc ${ }^{\mathrm{TM}}$ XRS + System (Bio-Rad, USA). Three independent experiments were performed.

\section{Proliferation assay}

For the cell proliferation assay, SAS cells were plated into a flat bottom 96 -well plate at $2 \times 10^{3}$ cells per well in triplicate with $100 \mu \mathrm{L}$ DMEM (HyClone, USA) the day before and then cells were exposed to different concentrations of EEA. Cell counting kit-8 reagent (CCK-8, China) was added into each well after incubation at $37^{\circ} \mathrm{C}$ in a $5 \% \mathrm{CO}_{2}$ atmosphere for $24,48,72,96$, and $120 \mathrm{~h}$. After incubation for $2 \mathrm{~h}$, absorbance at $450 \mathrm{~nm}$ was measured
Table 1. Antibodies used in the study.

\begin{tabular}{lrl}
\hline Antibody & Cat No. & Dilution \\
\hline GAPDH & 8848 & $1: 2000$ \\
Cleaved PARP & 5625 & $1: 1000$ \\
Cleaved caspase-9 & 9505 & $1: 1000$ \\
MMP-2 & 40994 & $1: 1000$ \\
MMP-9 & 13667 & $1: 1000$ \\
TIMP-1 & 8946 & $1: 1000$ \\
TIMP-2 & 5738 & $1: 1000$ \\
Anti-rabbit lgG, HRP-linked antibody & 7074 & $1: 500$ \\
Anti-mouse IgG, HRP-linked antibody & 7076 & $1: 500$ \\
\hline
\end{tabular}

MMP: matrix metalloproteinase; TIMP: tissue inhibitors of metalloproteinase.

using a microplate reader (Bio-Rad). Three independent experiments were performed.

\section{Transwell and wound healing assays}

A total of $4 \times 10^{4}$ SAS cells in $100 \mu \mathrm{L}$ serum-free DMEM medium (HyClone) supplemented with different concentrations of EEA were plated into the upper chamber of an $8-\mu \mathrm{m}$ pore membrane (Corning, USA) coated with matrigel (Becton, Dickinson and Company, USA) and $600 \mu \mathrm{L}$ complete medium was added as a chemoattractant to the lower chamber. After 36-h incubation, non-invading cells were scraped out with a cotton swab, membranes were then fixed with $100 \%$ methanol and stained with $0.5 \%$ crystal violet. Then, cells were photographed using a digital light microscope and five fields were selected randomly to evaluate the average cell number on each membrane. Three independent experiments were performed.

For the wound healing assay, $1 \times 10^{5}$ cells were seeded in 6 -well plates at $37^{\circ} \mathrm{C}$. When confluence reached $100 \%$, the monolayer of cells was scratched with a sterilized pipette tip so as to create a wound. Dead cells were then washed away with PBS and monolayer cells were treated with various concentrations of EEA in DMEM supplemented $10 \% \mathrm{FBS}$ at $37^{\circ} \mathrm{C}$ for $36 \mathrm{~h}$. Images were captured by a digital light microscope at 0 and $36 \mathrm{~h}$. Migration rate of cells at $36 \mathrm{~h}$ was indicated as wound width normalized to that at $0 \mathrm{~h}$. Three independent experiments were performed.

\section{Animal experiments}

All animal experiments were approved by the Animal Care and Use Committee of Fuyang Vocational Technical College (FVTC-20180621-031J-029). All procedures were in accordance with the Animal Care and Use Committee of the institution and conformed to legal mandates and national guidelines for the care and maintenance of laboratory animals. A total of 12 nude BALB/c mice (females, 6-8 weeks) were purchased from SLAC Laboratory Animal Co. Ltd. (China). SAS cells (10 million) were subcutaneously 
injected into the left flank of nude BALB/c mice. When tumors were palpable, all mice were randomized into 2 groups of 6 mice each. EEA stock solution was diluted to $20 \mathrm{mg} / \mathrm{mL}$ using normal saline for animal treatment. One group was orally administered diluted EEA solution at a dose of $100 \mathrm{mg} / \mathrm{kg}$ every other day for three weeks, and the other group was treated with an equal volume of solvent $(60 \%$ normal saline $+40 \%$ DMSO). Tumor volume was monitored every three days using a caliper. After 57 days, mice were sacrificed and tumors were excised. Tumor volume was calculated using the formula: volume $=$ $0.5 \times$ length $\times$ width $^{2}$.

\section{Statistical analysis}

All statistical analyses were performed using SPSS 19.0 (IBM, USA). Data are reported as means \pm SD. The $t$-test was used to compare the differences between experimental and control groups, while one-way ANOVA was used when more than two groups were compared. $\mathrm{P}<0.05$ was considered to indicate a statistically significant difference.

\section{Results}

\section{EEA effectively inhibited the proliferation of HNSCC cells}

We first assessed the effect of EEA on the proliferation capacity of HNSCC cells. Human tongue carcinoma cells (SAS) were treated with EEA ranging from 0.5 to $25 \mu \mathrm{g} / \mathrm{mL}$. After incubation for 5 days, cell proliferation was significantly inhibited by EEA ( 2.5 to $25 \mu \mathrm{g} / \mathrm{mL}$ ) compared to control, and a dose-dependent effect was also observed (Figure 1A). To further investigate the inherent molecular mechanisms mediating the inhibition effect of EEA on HNSCC cell proliferation, western blot analysis was conducted to determine the cellular levels of cleaved caspase9 and cleaved PARP, which were markedly increased in cells treated with EEA in a dose-dependent manner (Figure 1B). Increased cellular levels of cleaved caspase9 and cleaved PARP have been well-known as markers for the starting of cell apoptosis. Based on the above data, it can be hypothesized that EEA might effectively inhibit HNSCC cell proliferation potentially via provoking cell apoptosis.

\section{Inhibition of invasion and migration by EEA}

Results of wound healing assays indicated an average migration rate of $78.7 \%$ in the control group, while average migration rates were $65.3,40.0$, and $7.0 \%$ in groups treated with $0.5,12.5$, and $25 \mu \mathrm{g} / \mathrm{mL}$ EEA, respectively (Figure 2A and B). All these demonstrated that treatment of SAS with EEA for $36 \mathrm{~h}$ retarded cell migration ability in a dose-dependent manner. Data of transwell assay showed that, on average, 125 invasive cells per field were observed in control, and 72,54 , and 43 in the groups treated with 0.5 , 12.5 , and $25 \mu \mathrm{g} / \mathrm{mL}$ EEA, respectively (Figure $3 \mathrm{~A}$ and $\mathrm{B}$ ).
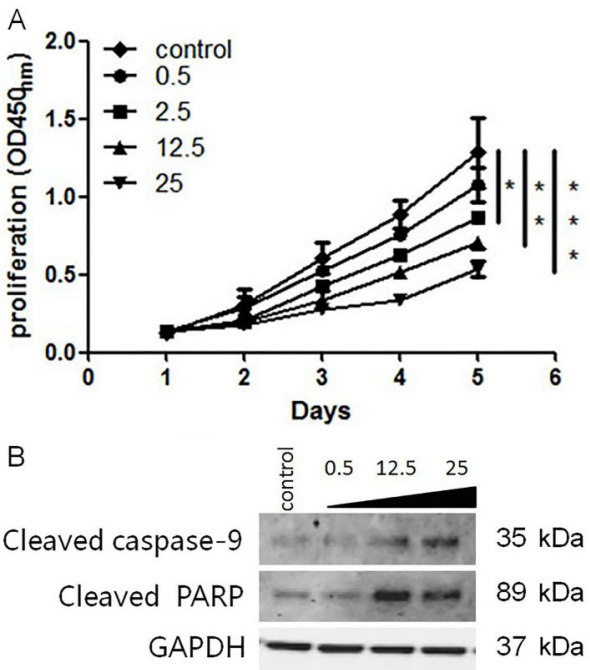

Figure 1. Ethanol extracts of Antrodia cinnamomea (EEA) inhibited cell proliferation in a dose-dependent manner. A, SAS cells were treated with EEA ranging from 0.5 to $25 \mu \mathrm{g} / \mathrm{m}$, and proliferation inhibition was significantly observed in cells treated with EEA over $2.5 \mu \mathrm{g} / \mathrm{mL}$ after incubation for 5 days. B, Cleaved caspase-9 and cleaved PARP were increased in cells treated with EEA. Data are reported as means $\pm S D$. ${ }^{*} P<0.05$, ${ }^{* *} P<0.01$, ${ }^{* * *} \mathrm{P}<0.001$ (ANOVA).

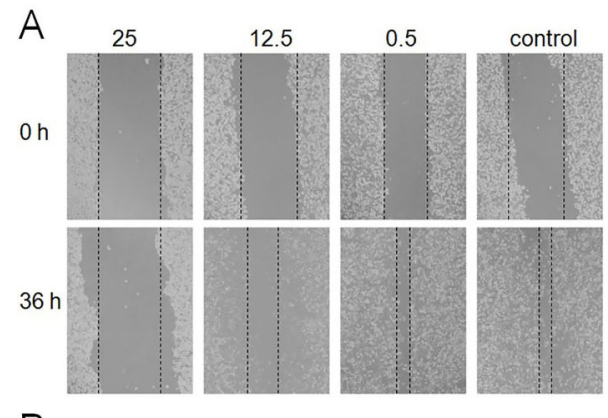

B

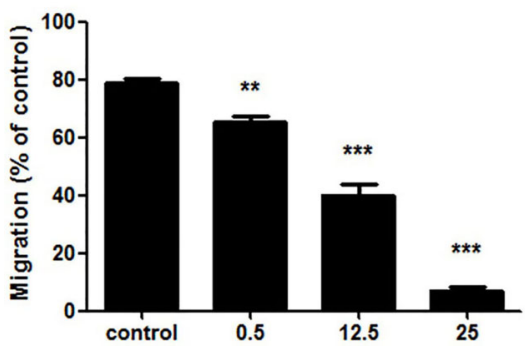

Figure 2. Suppression of wounding healing by ethanol extracts of Antrodia cinnamomea (EEA). A, A wound was made when cell confluence was $100 \%$ and fresh medium supplemented with different concentrations of EEA was added. Images were captured at 0 and 36 h. B, Migration distance was calculated and normalized to control that was not treated with EEA. Data are reported as means $\pm S D$. ${ }^{* *} \mathrm{P}<0.01,{ }^{* \star *} \mathrm{P}<0.001$ compared to control (ANOVA). 

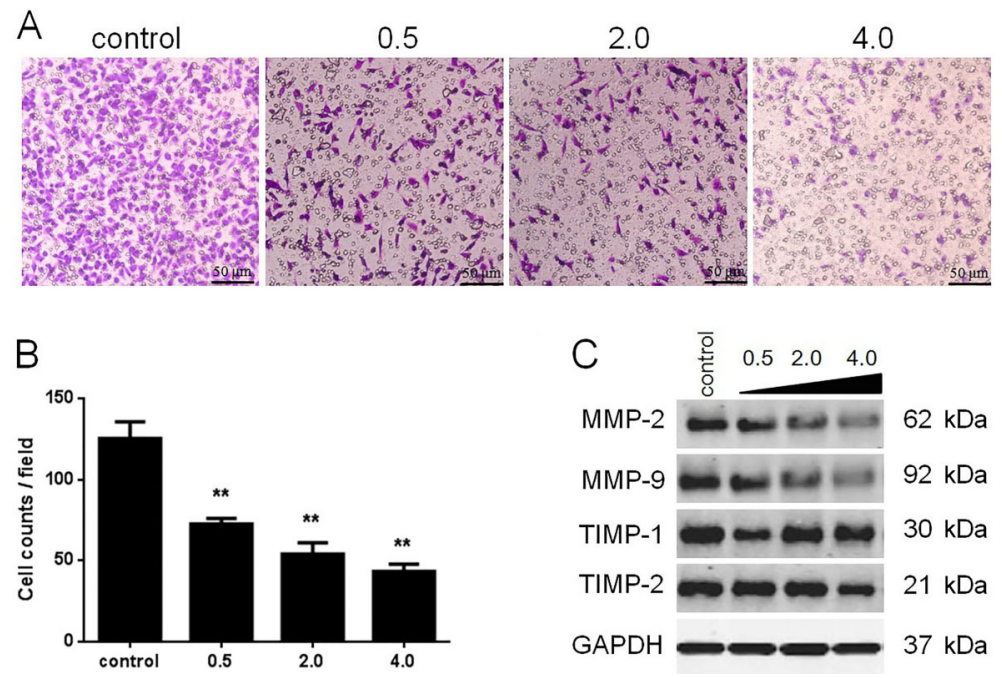

Figure 3. Inhibitive effects of ethanol extracts of Antrodia cinnamomea (EEA) on SAS cell invasion. A, SAS cells were plated into the upper chamber in medium supplemented with various concentrations of EEA. After 36-h incubation, invading cells were counted in five randomly selected fields (scale bars: $50 \mu \mathrm{m}$. B. The average counts of invading cells were significantly less in groups treated with EEA than in control. C, Effects of EEA on invasion-associated proteins, including MMP-2, MMP-9, TIMP-1, and TIMP-2, were evaluated by western blot. Data are reported as means $\pm \mathrm{SD}$. ${ }^{* \star} \mathrm{P}<0.01$ (ANOVA).
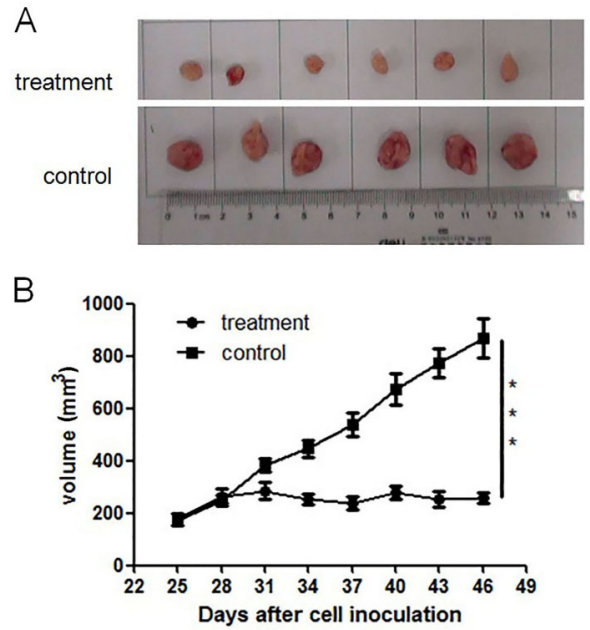

Figure 4. Inhibitive effects of ethanol extracts of Antrodia cinnamomea (EEA) on tumorigenesis in vivo. A and $\mathbf{B}$, Nude $B A L B / c$ mice were inoculated with SAS cells and treated with EEA when tumors were palpable. Tumor growth was significantly inhibited by EEA administration. Data are reported as means \pm SD. ${ }^{* * *} \mathrm{P}<0.001$ (ANOVA).

Transwell assay illustrated that the number of invasive cells was significantly reduced by EEA treatment in a concentration-dependent manner. Matrix metalloproteinases (MMPs) and tissue inhibitors of metalloproteinase (TIMP) are the most studied factors that directly influence the invasion and migration capacities of tumor cells.
As shown in Figure 3C, a dose-dependent decrease in expressions of MMP-2 and MMP-9 were observed in cells treated with EEA $(0.5$ to $25 \mu \mathrm{g} / \mathrm{mL})$, while TIMP-1 and TIMP-2 expressions were not affected. Based on the above data, it can be proposed that EEA was an effective inhibitor of migration and invasion of SAS partially by decreasing expression of MMP-2 and MMP-9.

\section{Anti-tumorigenic ability of EEA}

Based on the observed effects of EEA on SAS in vitro, we determined its impact on tumorigenesis in vivo. Tumor growth was significantly slowed down in mice treated by EEA compared to control (Figure 4A and 4B), indicating that EEA treatment significantly inhibited tumorigenesis in vivo.

\section{Discussion}

Head and neck squamous cell carcinoma is globally recognized as one of the most common malignancies with an increasing incidence rate $(3,18)$. Patients suffering from malignancies often experience recurrence or metastasis after treatments such as surgery, radiation therapy, chemotherapy, and targeted therapy $(19,20)$, and clinical outcomes remain unsatisfactory (21). Antrodia cinnamomea has been commonly used as food for its diverse benefits for health, including anti-inflammatory (15), antioxidant, liver protection (22), and anticancer (7). Here we explored the potential application of Antrodia cinnamomea in treatment of head and neck squamous cell carcinoma. 
Tumor cell proliferation and migration are mostly responsible for cancer progression. This study investigated the effects of EEA on proliferation and migration of HNSCC cells, and we found that EEA might inhibit proliferation and migration of SAS in a dose-dependent manner. Meanwhile, advanced experiments were conducted to briefly determine the mechanisms of the EEA-induced impact on SAS. We observed that cellular levels of cleaved caspase9 and cleaved PARP were upregulated in cells treated with increasing doses of EEA, implying that provoking cell apoptosis was one of the mechanisms mediating the effect of EEA on proliferation. Recent study demonstrated that EEA could induce cell cycle arrest of lung cancer cells, thus leading to proliferation inhibition (23).

Tumor metastasis is a complicated process involving diverse types of proteins, of which the most well studied are MMPs and TIMP. In our study, we found that MMP-2 and MMP-9 expressions were decreased by administration of EEA while TIMP-1 and TIMP-2 were not affected. A study focusing on lung cancer also reported that extracts of Antrodia cinnamomea decreased expression of MMP-2 and MMP-9 as well as their activities, which was ascribed to dysregulation of ERK, JNK, p38, and PI3K/Akt signaling pathways (7). A constituent isolated from Antrodia cinnamomea was confirmed to be an anti-metastatic compound mostly via reducing expression of MMP-2 and MMP-9 and enhancing E-cadherin and TIMP-1 expression (24).

Natural products extracted from plants or microorganisms are important sources for drug screening $(22,25)$. Several compounds extracted from fruit bodies of Antrodia cinnamomea have been found to efficiently influence tumor progression both in vivo and in vitro. For example, YMGKI-1, purified from natural products of Antrodia cinnamomea, was shown to effectively decrease cell viability of HNSCC cells and successfully inhibit tumorigenicity of

\section{References}

1. Chang CW, Chen YS, Chen CC, Chen CC, Sheu SJ, Lin TW, et al. Lyophilized particles and ethanolic extracts of Antrodia cinnamomea mycelia suppress the tumorigenicity of head and neck cancer cells in vivo. Biomedicine (Taipei) 2014; 4: 26.

2. Okamoto A, Chikamatsu K, Sakakura K, Hatsushika K, Takahashi G, Masuyama K. Expansion and characterization of cancer stem-like cells in squamous cell carcinoma of the head and neck. Oral Oncol 2009; 45: 633-639, doi: 10.1016/ j.oraloncology.2008.10.003.

3. Feldman R, Gatalica Z, Knezetic J, Reddy S, Nathan CA, Javadi $\mathrm{N}$, et al. Molecular profiling of head and neck squamous cell carcinoma. Head Neck 2016; 38: E1625E638, doi: 10.1002/hed.24290.

4. Koffler J, Sharma S, Hess J. Predictive value of epigenetic alterations in head and neck squamous cell carcinoma. Mol Cell Oncol 2014; 1: e954827, doi: 10.1080/23723548.2014. 954827.
SAS (14). Antcin-H, a type of triterpene isolated from Antrodia cinnamomea, was reported to inhibit proliferation and migration of human renal carcinoma $786-0$ cells by targeting MMPs and TIMPs (10). Furthermore, recent articles $(12,13,16)$ revealed that EEA enhances chemosensitivity of several carcinomas, indicating that EEA can be used as adjuvant treatment combined with conventional chemotherapy. Several investigations have already shown the synergistic effects of EEA combined with cisplatin (16), sorafenib (12), and tamoxifen (13). The antitumor effect of EEA has been confirmed in a randomized controlled study, although the synergistic effects of EEA combined with chemotherapy were not observed (26). It has been proposed that cancer stem-like cells were involved in tumor initiation and occurrence of chemoradiotherapy resistance, responsible for compromised clinical outcomes of conventional therapies $(2,27,28)$. Su et al. (17) reported that extracts of Antrodia cinnamomea sensitize cancer stem-like cells including HNSCC to chemoradiotherapy. Collectively, extracts of Antrodia cinnamomea are an important source for new drug discovery.

We conclude that this study comprehensively verified the inhibitive effects of EEA on cell proliferation, invasion, and migration of HNSCC in vitro and in vivo, and the potential molecular mechanism mediating the observed effects. These findings provide the basis for further study of the application of EEA as an effective candidate for cancer treatment.

\section{Acknowledgments}

This project was supported by Key Projects of Outstanding Young Talents support program in 2016 (gxyqZD 2016499) and Drug Production Technology Curriculum Group Teachers Teaching Innovation Team (2019 cxtd005).

5. Wilken R, Veena MS, Wang MB, Srivatsan ES. Curcumin: a review of anti-cancer properties and therapeutic activity in head and neck squamous cell carcinoma. Mol Cancer 2011; 10: 12, doi: 10.1186/1476-4598-10-12.

6. Metzler VM, Pritz C, Riml A, Romani A, Tuertscher R, Steinbichler T, et al. Separation of cell survival, growth, migration, and mesenchymal transdifferentiation effects of fibroblast secretome on tumor cells of head and neck squamous cell carcinoma. Tumor Biol 2017; 39: 10104283 17705507, doi: 10.1177/1010428317705507.

7. Chen YY, Liu FC, Chou PY, Chien YC, Chang WS, Huang GJ, et al. Ethanol extracts of fruiting bodies of Antrodia cinnamomea suppress CL1-5 human lung adenocarcinoma cells migration by inhibiting matrix metalloproteinase-2/9 through ERK, JNK, p38, and PI3K/Akt signaling pathways. Evid Based Complement Alternat Med 2012; 2012: 378415, doi: $10.1155 / 2012 / 378415$ 
8. Liu YM, Liu YK, Lan KL, Lee YW, Tsai TH, Chen YJ. Medicinal fungus Antrodia cinnamomea inhibits growth and cancer stem cell characteristics of hepatocellular carcinoma. Evid Based Complement Alternat Med 2013; 2013: 569737 , doi: 10.1155/2013/569737.

9. Chang CW, Chen YS, Chen CC, Chan IO, Chen CC, Sheu $\mathrm{SJ}$, et al. The medicinal fungus Antrodia cinnamomea regulates DNA repair and enhances the radiosensitivity of human esophageal cancer cells. Oncotarget 2016; 7: 16, doi: 10.2147/OTT.S96355.

10. Chiu KY, Chen TH, Wen CL, Lai JM, Cheng CC, Liu HC, et al. Antcin-H isolated from Antrodia cinnamomea inhibits renal cancer cell invasion partly through inactivation of FAKERK-C/EBP-beta/c-Fos-MMP-7 pathways. Evid Based Complement Alternat Med 2017; 2017: 5052870, doi: 10. 1155/2017/5052870.

11. Chen MC, Hsu WL, Chou TC. Anti-cachectic effect of Antrodia cinnamomea extract in lung tumor-bearing mice under chemotherapy. Oncotarget 2018; 9: 19584-19596, doi: 10.2147/OTT.S96355.

12. Wu WD, Chen PS, Omar HA, Arafa EA, Pan HW, Jeng J, et al. Antrodia cinnamomea boosts the anti-tumor activity of sorafenib in xenograft models of human hepatocellular carcinoma. Sci Rep 2018; 8: 12914.

13. Lin YS, Lin YY, Yang YH, Lin CL, Kuan FC, Lu CN, et al. Antrodia cinnamomea extract inhibits the proliferation of tamoxifen-resistant breast cancer cells through apoptosis and skp2/microRNAs pathway. BMC Complement Altern Med 2018; 18: 152.

14. Chang CW, Chen CC, Wu MJ, Chen YS, Chen CC, Sheu $\mathrm{SJ}$, et al. Active component of Antrodia cinnamomea mycelia targeting head and neck cancer initiating cells through exaggerated autophagic cell death. Evid Based Complement Alternat Med 2013; 2013: 946451, doi: 10.1155/2013/ 946451.

15. Zheng J, Jiao S, Li Q, Jia P, Yin H, Zhao X, et al. Antrodia cinnamomea oligosaccharides suppress lipopolysaccharide-induced inflammation through promoting O-GIcNAcylation and repressing p38/Akt phosphorylation. Molecules 2017; 23. pii: E51, doi: 10.3390/molecules23010051.

16. Huang TH, Chiu YH, Chan YL, Wang H, Li TL, Liu CY, et al. Antrodia cinnamomea alleviates cisplatin-induced hepatotoxicity and enhances chemo-sensitivity of line-1 lung carcinoma xenografted in BALB/CByJ mice. Oncotarget 2015; 6: 25741-25754, doi: 10.18632/oncotarget. 4348.

17. Su YK, Shih PH, Lee WH, Bamodu OA, Wu ATH, Huang $\mathrm{CC}$, et al. Antrodia cinnamomea sensitizes radio-/chemotherapy of cancer stem-like cells by modulating microRNA expression. J Ethnopharmacol 2017; 207: 47-56, doi: 10.1016/j.jep.2017.06.004
18. Kuang J, Zhao M, Li H, Dang W, Li W. Identification of potential therapeutic target genes and mechanisms in head and neck squamous cell carcinoma by bioinformatics analysis. Oncol Lett 2016; 11: 3009-3014, doi: 10.3892/ ol.2016.4358.

19. Mishra S. Orthodontic therapy for paediatric cancer survivors: a review. J Clin Diagn Res 2017; 11: ZE01-ZE04, doi: 10.7860/JCDR/2017/23916.9404.

20. Prakash J. Cancer-associated fibroblasts: perspectives in cancer therapy. Trends Cancer 2016; 2: 277-279, doi: 10.1016/j.trecan.2016.04.005.

21. Kolberg M, Holand M, Agesen TH, Brekke HR, Liestol K, Hall KS, et al. Survival meta-analyses for $>1800$ malignant peripheral nerve sheath tumor patients with and without neurofibromatosis type 1. Neuro Oncol 2013; 15: 135-147, doi: 10.1093/neuonc/nos287.

22. Ker YB, Peng CC, Chang WL, Chyau CC, Peng RY. Hepatoprotective bioactivity of the glycoprotein, antrodan, isolated from Antrodia cinnamomea mycelia. PLoS One 2014; 9: e93191, doi: 10.1371/journal.pone.0093191.

23. Wu CH, Liu FC, Pan CH, Lai MT, Lan SJ, Wu CH, et al. Suppression of cell growth, migration and drug resistance by ethanolic extract of Antrodia cinnamomea in human lung cancer A549 cells and C57BL/6J allograft tumor model. Int $J$ Mol Sci 2018; 19. pii: E791, doi: 10.3390/ijms19030791.

24. Lin CC, Chen CC, Kuo YH, Kuo JT, Senthil Kumar KJ, Wang SY. 2,3,5-Trimethoxy-4-cresol, an anti-metastatic constituent from the solid-state cultured mycelium of Antrodia cinnamomea and its mechanism. J Nat Med 2015; 69: 513-521, doi: 10.1007/s11418-015-0916-6.

25. Lin IY, Pan MH, Lai CS, Lin TT, Chen CT, Chung TS, et al. CCM111, the water extract of Antrodia cinnamomea, regulates immune-related activity through STAT3 and NF-kappaB pathways. Sci Rep 2017; 7: 4862, doi: 10.1038/s41598-01705072-y.

26. Tsai MY, Hung YC, Chen $Y H$, Chen $Y H$, Huang $Y C$, Kao $\mathrm{CW}$, et al. A preliminary randomised controlled study of short-term Antrodia cinnamomea treatment combined with chemotherapy for patients with advanced cancer. BMC Complement Altern Med 2016; 16: 322, doi: 10.1186/s129 06-016-1312-9.

27. Zhang $Q$, Shi S, Yen Y, Brown J, Ta JQ, Le AD. A subpopulation of $\mathrm{CD} 133(+)$ cancer stem-like cells characterized in human oral squamous cell carcinoma confer resistance to chemotherapy. Cancer Lett 2010; 289: 151-160, doi: 10.1016/j.canlet.2009.08.010.

28. Murase M, Kano M, Tsukahara T, Takahashi A, Torigoe T, Kawaguchi S, et al. Side population cells have the characteristics of cancer stem-like cells/cancer-initiating cells in bone sarcomas. Br J Cancer 2009; 101: 1425-1432, doi: 10.1038/sj.bjc.6605330. 